\title{
Acute Achilles tendon rupture after treatment with levofloxacin in a patient with giant cell arteritis
}

\author{
Małgorzata Stasiek, Piotr Głuszko \\ Clinic of Rheumatology, National Institute of Geriatrics, Rheumatology and Rehabilitation, Warsaw, Poland
}

\begin{abstract}
The authors report a case of a 67-year-old woman with giant cell arteritis with acute Achilles tendon rupture, which occurred after 3 days of levofloxacin therapy introduced because of newly diagnosed erosive gastritis associated with Helicobacter pylori infection. The Achilles tendon rupture was surgically treated and the patient made a complete recovery. In view of the widespread use of levofloxacin in practice, this case report raises important clinical implications. Tendinopathies are a known complication, quite rare in the healthy population, but the risk of rupture significantly increases in the population of patients over 60 years of age, with chronic usage of glucocorticosteroids, impaired renal function and recipients of organ transplants. What needs underlining, there are also described differences between individual fluoroquinolones as a cause of tendon damage in this group. Considering the widespread use of this group of drugs in patients, knowledge about the risk of adverse events including tendinopathy promotes safe use of fluoroquinolones.
\end{abstract}

Key words: fluoroquinolones, Achilles tendon, tendinopathy, rupture.

\section{Introduction}

Fluoroquinolones (FQ) are currently used as common antibacterial drugs administered because of broad antibacterial spectrum and good bioavailability when used orally. FQ are considered as safe and well tolerated, which is why they have been extensively distributed in outpatient care over the past 30 years. Despite their usefulness, FQ treatment may cause adverse events. There are many reports indicating that FQ can cause pathologic lesions in the tendon tissue from tendinopathy to rupture [1].

Some time ago the American Food and Drug Administration (FDA) as well as the European Medical Agency (EMA) decided to issue alerts regarding adverse events caused by this group of drugs. Despite the published alerts, the use of fluoroquinolones is abused in daily practice. Therefore we wish to recall adverse events in the musculoskeletal system and to indicate patients at higher risk. Most of the cases of rupture are associated with other risk factors such as older age, glucocorticoid use and rheumatic, vascular or renal diseases [2-4].
Diagnosis of autoinflammatory disease such as giant cell arteritis and chronic therapy with glucocorticosteroids are significant predisposing factors for Achilles tendinopathy including tendon rupture. It is necessary for physicians, especially rheumatologists and orthopedists, to know about this possible complication of FQ treatment as well as factors favoring it, so that the choice of antibiotic therapy is considered in the context of risk factors for acute and chronic tendon damage. And for prophylaxis one should consider the choice of an antibiotic from another group in patients with existing risk factors for this complication.

\section{A case report}

A 67-year-old woman diagnosed with giant cell arteritis in 2014, chronically treated with methylprednisolone $4 \mathrm{mg}$ daily in combination with methotrexate $20 \mathrm{mg}$ once a week, was referred for gastroscopy due to epigastric pain despite the use of a proton pump inhibitor. Gastroscopic examination revealed erosive gastritis with the presence of Helicobacter pylori infection. The patient 
was consulted by a general practitioner (GP) and ordered H. pylori eradication therapy with the following scheme: levofloxacin $2 \times 500 \mathrm{mg}$ per day for 7 consecutive days in combination with amoxicillin and a proton pump inhibitor. The third day following the initiation of therapy, suddenly, the patient presented instability and impairment in walking. A sharp pain appeared in the Achilles tendon area of the left foot with swelling of the distal limb.

The patient visited the GP once again and received advice to complete eradication therapy according to the previously recommended schedule. The symptoms presented, according to the GP's opinion, were considered as associated with rheumatic disease and the patient was instructed to report them to a rheumatologist, which was scheduled in three weeks. Therefore she continued FQ treatment for the next 3 weeks.

The consulting rheumatologist revealed a clear defect in the Achilles tendon on the left foot with a bulging muscle belly in the proximal calf consistent with a tendon rupture. A calf squeeze test revealed plantar flexion on the right but there was none on the left.

Based on the clinical picture and physical examination, the possibility of Achilles tendon rupture was suspected. However, due to great difficulty in walking, persistent pain and swelling of the limb, deep vein thrombosis in the lower extremity could not be ruled out. The patient was referred urgently to the hospital for further instructions. The ultrasound scan confirmed a total rupture of the Achilles tendon and deep vein thrombosis was excluded. X-ray of the left foot and ankle revealed only soft tissue swelling. The patient underwent orthopedic surgery and was discharged to start a rehabilitation program. After about 6 weeks the patient was able to walk without any assistance.

\section{Discussion}

Among all possible events associated with FQ treatment, those from the musculoskeletal system are the most commonly discussed in the literature [5].

Table I. Risk factors for tendinopathy during fluoroquinolone treatment $[1,8]$

\begin{tabular}{|l|}
\hline Risk factors \\
\hline Older age $(>60)$ \\
\hline Men $>$ women $(2: 1)[8]$ \\
\hline Glucocorticosteroid treatment \\
\hline Fluoroquinolone treatment duration \\
\hline Renal impairment \\
\hline Chronic lung disease \\
\hline Diabetes \\
\hline Hemodialysis \\
\hline Use of newer FQ \\
\hline
\end{tabular}

The first case of fluoroquinolone-related tendinopathy was described as early as in 1983. Since then, a number of reports indicated adverse events localized in the musculoskeletal system, from trivial ones such as arthralgia or myalgia to tendon ruptures or rhabdomyolysis. Most cases reported in the literature relate to tendon damage - inflammation, degeneration or rupture of the tendons. Several risk factors for tendinopathy have been identified, and the most important include: age over 60 years, long-term glucocorticoid therapy, sport activities, history of rheumatic diseases, diabetes, renal impairment and hemodialysis and organ transplants [5-8] (Table I).

Considering age and history of chronic rheumatic diseases treated with glucocorticoid therapy, which certainly affects processes of tendon repair, our patient was undoubtedly at risk of tendinopathy.

The most common symptom of FQ-associated tendinopathy is pain, which in our patient appeared on the third day of FQ treatment. This pain is usually of a sudden onset, and may be accompanied by acute signs of inflammation and swelling, which was presented as well. The diagnosis is made by acute/sub-acute onset of pain and swelling over the tendon, together with a history of recent use of $\mathrm{FQ}$ and the absence of other obvious causes of tendinopathy [3].

Clinical examination can reveal a "gap sign" but after a time edema can obliterate this area and palpation becomes unreliable. Thompson's test reveals failure of plantar flexion after compression of the gastrocnemius muscle in the patient lying in a prone position, which confirms tendon rupture. It is the best known and the easiest test, which was performed in our patient and was positive in the affected area. As many as $50 \%$ of reported cases manifested bilateral symptoms, but at the same time up to $50 \%$ of tendon rupture cases may not be preceded by any heraldic symptom $[5,7]$.

The Achilles tendon is most often affected (89.8\% of all cases), although other tendons may also be involved [9]. The weight-bearing role of the Achilles tendon is thought to be the reason for the high preponderance of injury in this structure $[10,11]$. Of all tendinopathies, up to $50 \%$ of patients may experience tendon rupture, of which almost $1 / 3$ of ruptures occur in patients receiving long-term treatment with glucocorticosteroids [7]. The time of onset of tendinopathy after FQ treatment can be very different, from the appearance of symptoms up to 2 hours after the first dose of the drug, with the highest frequency during the first month, to manifestations up to 6 months after stopping the drug [12].

In our patient tendon rupture occurred on the third day of treatment. According to other reports the average time of rupture onset after use of FQ is 8 to 14 days [13]. The incidence rate for tendinopathy is $0.1 \%$ to $0.01 \%$, and the incidence rate for tendon rupture is 
less than 0.01\% [14]. Van der Linden et al. [15] estimate that between 2 and $6 \%$ of all Achilles tendon ruptures in patients over 60 years of age can be attributed to the use of FQ. According to Corrao et al. [16], patients over 60 years of age treated with FQ from 1 to 30 days have a 1.5-2.7 times higher risk of tendon injury including rupture compared to younger patients (under 60 years of age). Some reviews indicate that men are more likely to develop rupture $[3,12]$.

Some authors believe that the different chemical structure of newer FQ (levofloxacin, ofloxacin and pefloxacin) makes them more toxic for cartilages and tendons than the older group of FQ (norfloxacin, ciprofloxacin and enoxacin) [17, 18]. The underlying pathophysiology of FQ-induced tendinopathy is not entirely known, although several concepts are suggested. Changes in tendons and their damage are associated with, among other things, chemical properties such as chelating abilities of fluoroquinolones. There was also proposed the hypothesis about molecular mechanisms such as inhibition of cellular proliferation in tendons, inhibition of tenocyte migration and increased expression of matrix metalloproteinase that induces collagen degradation and some others [19].

Fluoroquinolone-induced tendinopathies occur at recommended doses and treatment duration [10]. However, the severity of tendinopathy appears to be proportional to the treatment duration [10]. The diagnosis is usually clinical, although the use of imaging methods such as ultrasound or magnetic resonance imaging can be helpful. Typical US findings of tendinopathy are thickened tendon with increased blood flow seen in color Doppler examination [3]. Magnetic resonance imaging can be clinically helpful especially in the presence of questionable diagnosis or for tear localization during presurgical planning [20].

Management of FQ-induced tendinopathy is largely symptomatic - withdrawal of harmful, suspected drug, relief, ice packs, use of painkillers and physiotherapy. In the case of almost complete or complete rupture surgery is necessary to restore tendon continuity. Even with early diagnosis and appropriate management, ten- dinopathy heals slowly. The mean recovery time ranges from 3 weeks to 3 months [10]. Early and prolonged physical therapy is frequently required, particularly for elderly patients [10]. However, the most important is prevention of rupture, as most patients recover within 2 months of stopping therapy, but more than a quarter of them may suffer from persistent pain and disability [5].

In the present case report, the recommendation was to use levofloxacin as the first line therapy for eradication of $\mathrm{H}$. pylori infection, but it is not the best first line treatment option for the described patient. Fluoroquinolones are a very effective group of antibiotics but current recommendations regarding the use of this group of antibiotics, after alerts of the FDA and EMA, should be narrowed to the justified medical situations listed in Table II. It is important because FQ can cause not only tendinopathy but also a prolonged (up to months or years), serious, disabling and potentially irreversible syndrome of symptoms from multiple systems of organs and senses (FQAD - long-term fluoroquinolone-associated disability) whose prevalence is probably underestimated because it mimics other diseases.

The clinical picture of FQAD includes tendinopathy, muscle weakness, peripheral polyneuropathy, autonomic nervous system dysfunction, sleep disorders, psychological symptoms, and even complete impairment of function and consciousness [21]. Especially, this group of drugs should not be used in a case of previous history of FQ-related tendinopathy, and should be avoided in elderly patients with chronic glucocorticoid use. According to the most recent meta-analysis, increase in risk sizes is between 4.68-fold of Achilles tendon rupture and 14.72-fold of Achilles tendinitis for patients simultaneously using FQ and glucocorticosteroids, compared with the initial estimates [22].

Considering the frequent, chronic use of glucocorticosteroids and the inflammatory and destructive involvement of tendons in rheumatic diseases, and the growing proportion of the elderly in the general population in Poland and other European countries, FQ should not be considered as first choice drugs in antimicrobial therapies.

Table II. Disabling and potentially permanent adverse events lead to suspension or restrictions of quinolone and fluoroquinolone administration [www.ema.europa.eu]

\begin{tabular}{|l|}
\hline Restrictions on the use of fluoroquinolone antibiotics mean that they should NOT be used \\
\hline To treat infections that might get better without treatment or are not severe (such as throat infections) \\
\hline To treat non-bacterial infections, e.g. non-bacterial (chronic) prostatitis \\
\hline $\begin{array}{l}\text { For preventing traveler's diarrhea or recurring lower urinary tract infections (urine infections that do not extend beyond } \\
\text { the bladder) }\end{array}$ \\
$\begin{array}{l}\text { To treat mild or moderate bacterial infections unless other antibacterial medicines commonly recommended for these } \\
\text { infections cannot be used }\end{array}$ \\
\hline
\end{tabular}


Currently there are many other better and potentially safer therapeutic alternatives. However, if there is no better option, patients should be informed about possible side effects of the drug and they should refer all those suspected of tendinitis symptoms to the doctor. The severity of tendinopathy appears to be proportional to duration of the treatment [10] so early intervention as discontinuing the drug immediately gives the patient the chance to minimize side effects. A clear temporal relationship can be observed between the implementation of FQ and the onset of symptoms, which considerably increases the suspicion of drug-induced damage in differential diagnosis in our patient. Currently, due to the lack of effective conservative treatment, the surgical procedures and subsequent rehabilitation have remained the basic way of management in tendon damage after FQ treatment. Hence we remain prudent in using these drugs, with particular emphasis on the treatment in the patients in the risk groups for these complications, and conduct strict monitoring of patients during FQ use.

\section{Conclusions}

Levofloxacin-induced tendinopathy and/or tendon rupture are uncommon but well-known complications. However, sometimes symptoms in association with risk factors such as older age and usage of glucocorticoids and damage such as a rupture may require surgery, may also cause irreversible disability. Therefore prevention which consists in avoiding the use of drugs in risk groups and obeying the rules according to the recommendations for subsequent lines of antibacterial therapy are vitally important.

The authors declare no conflict of interest.

\section{References}

1. Lewis T, Cook J. Fluoroquinolones and tendinopathy: a guide for athletes and sports clinicians and a systematic review of the literature. J Athlet Train 2014; 49: 422-427.

2. Morandé SC, de Rada Lorente PD, Clemente JD, et al. Bilateral Achilles tendon rupture secondary to levofloxacin. Rev Esp Cir Ortop Traumatol (English Edition) 2010; 54: 238-240.

3. Tam PK, Ho CT. Fluoroquinolone-induced Achilles tendinitis. Hong Kong Med J 2014; 20: 545-547.

4. Garg S, Thilagarajah M. Bilateral rupture of Achilles tendon (bilrat) without predisposing systemic disease or steroid use: a case report and review of the literature. Int J Orthop Surg 2008; 13: 1-3.

5. Hal MM, Finnoff JT, Smith J. Musculoskeletal complications of fluoroquinolones: guidelines and precautions for usage in the athletic population. PM R 2011; 3: 132-142.
6. Emmerson AM, Jones AM. The quinolones: Decades of development and use. J Antimicrob Chemother 2003; 51 (Suppl. 1): 13-20.

7. US Food and Drug Administration. Information for Healthcare Professionals: Fluoroquinolone Antimicrobial Drugs. http:// www.fda.gov/Drugs/DrugSafety/PostmarketDrugSafetylnformationforPatientsandProviders/ucm 126085.htm

8. Kim GK. The Risk of Fluoroquinolone-induced Tendinopathy and Tendon Rupture: What Does The Clinician Need To Know? J Clin Aesthet Dermatol 2010; 3: 49-54.

9. Yu C, Guiffre BM. Achilles tendinopathy after treatment with fluoroquinolone. Australas Radiol 2005; 49: 407-410.

10. Connelly S, Bayliff C, Mehta S. Levofloxacin-induced bilateral Achilles tendinopathy. Canad J Hosp Pharm 2002; 55: 212-214.

11. Greene BL. Physical therapist management of fluoroquinolone-induced Achilles tendinopathy. Phys Ther 2002; 82: 12241231

12. Khaliq Y, Zhanel GG. Fluoroquinolone-associated tendinopathy: a critical review of the literature. Clin Infect Dis 2003; 36: 1404-1410.

13.Fernández-Cuadros ME, Casique-Bocanegra LO, AlbaladejoFlorín MJ, et al. Bilateral Levofloxacin-Induced Achilles Tendon Rupture: An Uncommon Case Report and Review of the Literature. Clin Med Insights Arthritis Musculoskelet Disord 2019; 12: 1179544119835222.

14. Haddow LJ, Chandra Sekhar M, Hajela V, Gopal Rao G. Spontaneous Achilles tendon rupture in patients treated with levofloxacin. J Antimicrob Chemother 2003; 51: 747-748.

15.van der Linden PD, Sturkenboom MC, Herings RM, et al. Increased risk of Achilles tendon rupture with quinolone antibacterial use, especially in elderly patients taking oral corticosteroids. Arch Intern Med 2003; 163: 1801-1807.

16. Corrao G, Zambon A, Bertù L, et al. Evidence of tendinitis provoked by fluoroquinolone treatment: a case control study. Drug Saf 2006; 29: 889-896.

17. Lado Lado FL, Rodríguez Moreno C, Velasco González M, et al. Rotura parcial bilateral aquílea asociada a levofloxacino. An Med Interna (Madrid) 2005; 22: 28-30.

18. Jeffrey K. Aronson JK (ed.). Meyler's side effects of drugs. $16^{\text {th }}$ ed. Elsevier Science, Amsterdam 2016.

19. Tsai WC, Yang YM. Fluoroquinolone-associated tendinopathy. Chang Gung Med I 2011; 34: 461-467.

20. Ramirez MA, Richardson LC. Pulmonary embolism associated with spontaneous bilateral Achilles tendon rupture. J Foot Ankle Surg 2007; 46: 283-287.

21. Golomb BA, Koslik HJ, Redd AJ. Fluoroquinolone-induced serious, persistent, multisymptom adverse effects. BMJ Case Rep 2015; 2015: pii: bcr2015209821.

22. Alves C, Mendes D, Marques FB. Fluoroquinolones and the risk of tendon injury: a systematic review and meta-analysis. Eur J Clin Pharmacol 2019; 75; 1431-1443. 